\title{
ON RINGS OF SUPERSYMMETRIC POLYNOMIALS
}

\author{
A.N. SERGEEV
}

\begin{abstract}
We consider three types of rings of supersymmetric polynomials: polynomial ones $\Lambda_{m, n}$, partially polynomial $\Lambda_{m, n}^{+y}$ and Laurent supersymmetric rings $\Lambda_{m, n}^{ \pm}$. For each type of rings we give their descriptions in terms of generators and relations. As a corollary we get for $n \geq m$ an isomorphism $\Lambda_{m, n}^{+y}=\Lambda_{m, m}^{+y} \otimes \Lambda_{0, n-m}^{+y}$. It is also true for polynomial rings, but in this case the isomorphism does not preserve the grading. For each type of rings some natural basis consisting of Euler supercharacters is constructed.
\end{abstract}

\section{Contents}

1. Introduction

2. Laurent symmetric polynomials

3. Supersymmetric partially polynomial and polynomial rings 9

4. Laurent supersymmetric polynomials 15

5. Jacobi-Trudy formulae and Euler supercharacters 18

6. Example

7. Acnowledgements 25

References

\section{INTRODUCTION}

Rings of symmetric polynomials $\Lambda_{m}$ play an important role in many areas of mathematics (see [9]). From the representation theory point of view ring $\Lambda_{m}=\mathbb{Z}\left[x_{1}, \ldots, x_{m}\right]^{S_{m}}$ is the Grothendieck ring of polynomial finite dimensional representations of the algebraic group $G L(m)$. The ring $\Lambda_{m}$ has a natural linear basis consisting of the classes of irreducible polynomial representations. Corresponding symmetric polynomials are known as Schur polynomials. They can be expressed by the Weyl formula or the JacobiTrudy formula. It is also well known that the ring $\Lambda_{m}$ is freely generated by elementary symmetric polynomials $e_{1}, \ldots, e_{m}$ as well as complete symmetric polynomials $h_{1}, \ldots, h_{m}$ and both families are algebraically independent. So the problem of describing this ring in terms of generators and relations is trivial in this case.

Key words and phrases. Supersymmetric polynomials, Generators and relations, Lie superalgebras, Euler supercharacters. 
If we consider all finite dimensional representation of the algebraic group $G L(m)$ then the corresponding ring is the ring of symmetric Laurent polynomials (see for example [3]) $\Lambda_{m}^{ \pm}=\mathbb{C}\left[x_{1}^{ \pm 1}, \ldots, x_{m}^{ \pm 1}\right]^{S_{m}}$. The ring $\Lambda_{m}^{ \pm}$has not been studied in such details as the polynomial one. It has of course a natural liner basis consisting of characters of irreducible finite dimensional representations which can be given again by the Weyl formula. There is also some analogue of the Jacobi-Trudy formula, which is called composite Schur functions $([2,10])$. It is also well known that $\Lambda_{m}^{ \pm}=\Lambda_{m}\left[e_{m}^{-1}\right]$. And a natural question is: How to describe this ring in terms of complete symmetric functions? It turns out, that an answer to this question can be naturally given in terms of Euler characters $H_{k}\left(x_{1}, \ldots, x_{n}\right), k \in \mathbb{Z}$

$$
H_{k}=E\left(k \varepsilon_{1}\right)=\frac{\left\{x_{1}^{k+m-1} x_{2}^{m-2} \ldots x_{m}^{0}\right\}}{\left\{x_{1}^{m-1} x_{2}^{m-2} \ldots x_{m}^{0}\right\}}
$$

where \{\} means the alternation on symmetric group $S_{m}$. It is easy to check, that $H_{k}=h_{k}-h_{k}^{\infty}, k \in \mathbb{Z}$ where $h_{k}, h_{k}^{\infty}$ are coefficients of Laurent series at zero and infinity of the function $\frac{1}{\Pi\left(1-x_{i} t\right)}$. It looks like these polynomials play the same role for Laurent symmetric polynomials as polynomials $h_{k}$ for usual symmetric polynomials. For example Jacobi-Trudy identity in this case can be written in the usual form

$$
E_{\lambda}\left(x_{1}, \ldots, x_{m}\right)=\operatorname{det}\left(H_{\lambda_{i}-i+j}\right)_{1 \leq i, j \leq m}
$$

Polynomials $H_{k}$ also allow to give a description of the algebra $\Lambda_{m}^{ \pm}$in terms of generators $h_{1}, h_{1}^{*}, \ldots$ and relations between them.

A remarkable feature of this approach is that it works in the same manner for rings of supersymmetric polynomials of all types as well. And it turns out that in supersymmetric case we need to consider function $\frac{\prod\left(1-y_{j} t\right)}{\prod\left(1-x_{i} t\right)}$ and define $H_{k}=h_{k}-h_{k}^{\infty}, k \in \mathbb{Z}$ by the same formula as before. Then relations for rings of supersymmetric polynomials (of all types) can be obtained by the same formulae as in $\Lambda_{m}^{ \pm}$case simply replacing number $m$ by the superdimension $m-n$. We should say that the importance of considering Laurent decomposition at infinity was first observed in the paper [8], and some of the relations for algebras supersymmetric polynomials $\Lambda_{m, n}$ were introduced in the paper [7].

As it was proved in [13] the ring $\Lambda_{m, n}^{ \pm}$is the quotient of the Grothendieck ring of the category finite dimensional representations of Lie supergroup $G L(m, n)$ by the relation $[M]=-[\Pi M]$ and it has a natural basis consisting of the classes irreducible finite dimensional representations. But there is no simple explicit formula for them [12], [1. So instead of characters of irreducible modules we use Euler supercharacters following explicit formula by Serganova [5] (We should note that Euler supercharacters can be easily obtained from the corresponding Euler characters). We also prove JacobiTrudy identity for Euler characters. One of the main technical tool in this paper is the evaluation homomorphism $\varphi: \Lambda_{m, n}^{ \pm} \rightarrow \Lambda_{m-1, n-1}^{ \pm}$such that 
$\varphi\left(x_{m}\right)=\varphi\left(y_{n}\right)$. It has been shown recently [6], that this evaluation homomorphism can be naturally induced by Duflo-Serganova functor. It would be interesting to give similar interpretation to other results of the present paper, for example Corollary 3.14 and Corollary 3.15 which state that for $n \geq m$ we have an isomorphisms $\Lambda_{m, n}^{+y}=\Lambda_{m, m}^{+y} \otimes \Lambda_{0, n-m}^{+y}$ and $\Lambda_{m, n}=\Lambda_{m, m} \otimes \Lambda_{0, n-m}$.

\section{LAURENT SYMMETRIC POLYNOMIALS}

In this section we are going to generalise some of the facts about symmetric polynomials to the case of Laurent symmetric polynomials. The main result of this section is a description of the rings of Laurent polynomials in terms of generators and relations. This problem is trivial for rings of usual symmetric polynomials, but it is not trivial for the rings of Laurent symmetric polynomials.

Definition 2.1. Let us define

$$
\Lambda_{m}^{ \pm}=\mathbb{Z}\left[x_{1}^{ \pm 1}, \ldots, x_{m}^{ \pm 1}\right]^{S_{m}}
$$

and we shall call this ring as the ring of Laurent symmetric polynomials.

Definition 2.2. Let $\lambda_{1}, \ldots, \lambda_{m}$ be any sequence of integers. Let us define Euler character $E_{\lambda} \in \Lambda^{ \pm}$by the following formula

$$
E_{\lambda}(x) \Delta_{m}(x)=\left\{E_{\lambda}(x) x_{1}^{m-1} \ldots, x_{m}^{0}\right\}=\left\{x_{1}^{\lambda_{1}+m-1} \ldots x_{m}^{\lambda_{m}}\right\}
$$

where $\Delta_{m}(x)=\prod_{i<j}\left(x_{i}-x_{j}\right)$ ra and $\{f(x)\}$ means alternation over group $S_{m}$

$$
\{f(x)\}=\sum_{\sigma \in S_{m}} \varepsilon(\sigma) \sigma(f),
$$

Theorem 2.3. Let $\lambda_{1} \geq \cdots \geq \lambda_{m}$ be integers, then $E_{\lambda}(x)$ form a basis of the ring $\Lambda_{m}^{ \pm}$.

Proof. If $\lambda_{1} \geq \cdots \geq \lambda_{m}$ then polynomials $\left\{x_{1}^{\lambda_{1}+m-1} \ldots x_{m}^{\lambda_{m}}\right\}$ form a basis in the space of Laurent skew-symmetric polynomials. Multiplication by $\Delta_{m}$ gives an isomorphism between vector space of symmetric polynomials and vector space of skew-symmetric polynomials.

Definition 2.4. For any integer $k$ let us set $H_{k}=E_{(k, 0, \ldots, 0)}$.

Let us also define polynomials $h_{k}, h_{k}^{(\infty)}$ by equalities

$$
\frac{1}{\prod_{i=1}^{m}\left(1-x_{i} t\right)}=\sum_{k=0}^{\infty} h_{k} t^{k}=\sum_{k=-\infty}^{-m} h_{k}^{(\infty)} t^{k}
$$

We as usually suppose that $h_{k}^{*}=h_{k}=0$ if $k<0$, where $h_{k}^{*}=h_{k}\left(x_{1}^{-1}, \ldots, x_{m}^{-1}\right)$.

It is easy to check that

$$
h_{k}^{(\infty)}=(-1)^{m}\left(x_{1} \ldots x_{m}\right)^{-1} h_{-m-k}^{*},
$$


Lemma 2.5. We have the following equalities

1)

$$
H_{k}=\left\{\begin{array}{l}
h_{k}, k \geq 0 \\
0,-m<k<0 \quad, \quad \text { or } \quad H_{k}=h_{k}-h_{k}^{(\infty)} \\
-h_{k}^{(\infty)} k \leq-m
\end{array}\right.
$$

2) $H_{k}-x_{1} H_{k-1}=H_{k}\left(x_{2}, \ldots, x_{m}\right)$

3) If $m=1$, then $H_{k}\left(x_{1}\right)-x_{1} H_{k-1}\left(x_{1}\right)=0$

Proof. To prove the first equality let us calculate the following generating function

$$
H_{m}^{+}(t)=\sum_{k=0}^{\infty} H_{k} t^{k}
$$

We have

$$
\begin{gathered}
H_{m}^{+}(t) \Delta_{m}(x)=\sum_{k=0}^{\infty}\left\{x_{1}^{k} t^{k} x_{1}^{m-1}, \ldots, x_{m}^{0}\right\}=\sum_{k=0}^{\infty}\left\{\frac{1}{1-x_{1} t} x_{1}^{m-1}, \ldots, x_{m}^{0}\right\} \\
=\frac{x_{1}^{m-1} \Delta^{(1)}(x)}{1-x_{1} t}-\frac{x_{2}^{m-1} \Delta^{(2)}(x)}{1-x_{2} t}-\cdots-\frac{x_{m}^{m-1} \Delta^{(m)}(x)}{1-x_{m} t}
\end{gathered}
$$

where

$$
\Delta^{(l)}(x)=\prod_{i, j \neq l, i<j}\left(x_{i}-x_{j}\right)
$$

On the other hand we have the decomposition in partial fractions

$$
\begin{gathered}
\frac{1}{\prod_{i=1}^{m}\left(1-x_{i} t\right)}=\frac{x_{1}^{m-1}}{\prod_{j \neq 1}\left(x_{1}-x_{j}\right)} \frac{1}{1-x_{1} t} \\
+\frac{x_{2}^{m-1}}{\prod_{j \neq 2}\left(x_{2}-x_{j}\right)} \frac{1}{1-x_{2} t}+\cdots+\frac{x_{m}^{m-1}}{\prod_{j \neq m}\left(x_{m}-x_{j}\right)} \frac{1}{1-x_{m} t}
\end{gathered}
$$

Therefore

$$
H_{m}^{+}(t)=\frac{1}{\prod_{i=1}^{m}\left(1-x_{i} t\right)}
$$

Now let us calculate generating function

$$
H_{m}^{-}(t)=\sum_{k=-1}^{-\infty} H_{k} t^{k}
$$

We have

$$
H_{m}^{-}(t) \Delta(x)=\sum_{k=1}^{\infty}\left\{x_{1}^{-k} t^{-k} x_{1}^{m-1}, \ldots, x_{m}^{0}\right\}=\sum_{k=0}^{\infty}\left\{\frac{x_{1}^{-1} t^{-1}}{1-x_{1}^{-1} t^{-1}} x_{1}^{m-1}, \ldots, x_{m}^{0}\right\}
$$

So $H_{m}^{-}(t)=-H_{m}^{+}(t)$. This proves the first statement.

Now let us prove the statement 2). It is enough to prove it separately for $h_{k}$ and $h_{k}^{(\infty)}$. We have $\left(1-x_{1} t\right) H_{m}^{+}(t)=H_{m-1}^{+}(t)$ So the statement 2$)$ is true for $h_{k}$. The case $h_{k}^{(\infty)}$ can be proved in the same way. 
It it easy to check that if $m=1$, then $H_{k}(x)=x^{k}, k \in \mathbb{Z}$ and the statement 3) follows.

Theorem 2.6. 1) Let $\lambda_{1}, \ldots, \lambda_{m}$ be any sequence of integers, then

$$
E_{\lambda}\left(x_{1}, \ldots, x_{m}\right)=\operatorname{det}\left(H_{\lambda_{i}-i+j}\right)_{1 \leq i, j \leq m}
$$

2) If $\lambda_{1}, \ldots, \lambda_{m+1}$ any sequence of integers, then we have the following equality in the ring $\Lambda_{m}^{ \pm}$

$$
\operatorname{det}\left(H_{\lambda_{i}-i+j}\right)_{1 \leq i, j \leq m+1}=0
$$

Proof. To prove the first statement we will use induction on $m$. If $m=1$, then $E_{k}(x)=H_{k}(x)$ by definition and the proof is trivial. Let $m>1$. Let us denote by $F_{\lambda}\left(x_{1}, \ldots, x_{m}\right)$ the determinant

$$
\left|\begin{array}{cccc}
H_{\lambda_{1}} & H_{\lambda_{1}+1} & \ldots & H_{\lambda_{1}+m-1} \\
\vdots & \vdots & \ddots & \vdots \\
H_{\lambda_{m}-m+1} & H_{\lambda_{m}-m+2} & \ldots & H_{\lambda_{m}}
\end{array}\right|
$$

Then we have

$$
F_{\lambda}\left(x_{1}, \ldots, x_{m}\right) \Delta_{m}(x)=\left|\begin{array}{cccc}
H_{\lambda_{1}} \Delta_{m}(x) & H_{\lambda_{1}+1} \Delta_{m}(x) & \ldots & H_{\lambda_{1}+m-1} \Delta_{m}(x) \\
\vdots & \vdots & \ddots & \vdots \\
H_{\lambda_{m}-m+1} & H_{\lambda_{m}-m+2} & \ldots & H_{\lambda_{m}}
\end{array}\right|
$$

Since $H_{k} \Delta_{m}(x)=\left\{x_{1}^{k+m-1} x_{2}^{m-2} \ldots x_{m}^{0}\right\}$ we get

$$
F_{\lambda}\left(x_{1}, \ldots, x_{m}\right) \Delta_{m}(x)=\left\{\mid \begin{array}{cccc}
x_{1}^{\lambda_{1}} & x_{1}^{\lambda_{1}+1} & \ldots & x_{1}^{\lambda_{1}+m-1} \\
H_{\lambda_{2}-1} & H_{\lambda_{2}} & \ldots & H_{\lambda_{2}+m-2} \\
\vdots & \vdots & \ddots & \vdots \\
H_{\lambda_{m}-m+1} & H_{\lambda_{m}-m+2} & \ldots & H_{1}^{m-1} \ldots x_{m}^{0}
\end{array}\right\}
$$

Now let us multiply every column (except the last one) by $x_{1}$ and subtract the result from the following column. Then we can apply formula 2) from Lemma 2.5 and expand the determinant along the first row. Then we get

$$
F_{\lambda}\left(x_{1}, \ldots, x_{m}\right) \Delta_{m}(x)=\left\{F_{\mu}\left(x_{2}, \ldots, x_{m}\right) x_{1}^{\lambda_{1}+m-1} x_{2}^{m-2} \ldots x_{m}^{0}\right\}
$$

where $\mu=\left(\lambda_{2}, \ldots, \lambda_{m}\right)$ and we can apply inductive assumption.

Let us prove the second statement also induction by $m$. If $m=1$, then for any integers $\lambda_{1}, \lambda_{2}$ we have

$$
\left|\begin{array}{cc}
H_{\lambda_{1}} & H_{\lambda_{1}+1} \\
H_{\lambda_{2}-1} & H_{\lambda_{2}}
\end{array}\right|=\left|\begin{array}{cc}
x^{\lambda_{1}} & x^{\lambda_{1}+1} \\
x^{\lambda_{2}-1} & x^{\lambda_{2}}
\end{array}\right|=0
$$

If $m>1$, then we can use the same arguments as before and induction.

Remark 2.7. It is easy to check, that if $l \leq m$, then

$$
\operatorname{det}\left(H_{\lambda_{i}-i+j}\right)_{1 \leq i, j \leq m}=\operatorname{det}\left(H_{\lambda_{i}-i+j}\right)_{1 \leq i, j \leq l}
$$

where $\lambda_{l+1}=\cdots=\lambda_{m}=0$. 
Corollary 2.8. Polynomials $H_{k}, k \in \mathbb{Z}$ generate the ring $\Lambda_{m}^{ \pm}$.

There exists one more formula of Jacobi-Trudy type for polynomials $E_{\lambda}$. It is called composite Schur functions [2, 10].

Lemma 2.9. Let $\lambda=\left(\lambda_{1}, \ldots, \lambda_{m}\right)$ be non-increasing sequence of integers. Let us write it in the form

$$
\left(\lambda_{1}, \ldots, \lambda_{m}\right)=\left(\mu_{1}, \ldots, \mu_{r}, 0, \ldots, 0,-\nu_{s}, \ldots,-\nu_{1}\right)
$$

where, $\mu, \nu$ are some partitions of the length $r u s$ correspondently. Then the following equality is valid

$$
E_{\lambda}=\left|\begin{array}{cccc}
h_{\nu_{s}}^{*} & h_{\nu_{s}-1}^{*} & \ldots & h_{\nu_{s}-s-r+1}^{*} \\
\vdots & \vdots & \ddots & \vdots \\
h_{\nu_{1}+s-1}^{*} & h_{\nu_{1}+s-2}^{*} & \ldots & h_{\nu_{1}-r}^{*} \\
h_{\mu_{1}-s} & h_{\mu_{1}-s+1} & \ldots & h_{\mu_{1}+r-1} \\
\vdots & \vdots & \ddots & \vdots \\
h_{\mu_{r}-s-r+1} & h_{\mu_{r}-s-r+2} & \ldots & h_{\mu_{r}}
\end{array}\right|
$$

Proof. It is easy to see that we can suppose that $r+s=m$, considering patritions with zero parts. Since the determinant has size $m \times m$, then by Lemma 2.5 the following equalities are valid $h_{i}=H_{i}, h_{j}^{*}=H_{-m}^{-1} H_{-m-j}$ for all elements of the determinant. So we can bring $H_{-m}^{-s}$ in front of the determinant and take into account that $H_{-m}^{-1}=(-1)^{m+1} x_{1} \ldots x_{m}$. Then after reordering rows and taking sings into account we get

$$
E_{\lambda}=\left(x_{1} \ldots x_{m}\right)^{s}\left|\begin{array}{cccc}
H_{\mu_{1}-s} & H_{\mu_{1}-s+1} & \ldots & H_{\mu_{1}+r-1} \\
\vdots & \vdots & \ddots & \vdots \\
H_{\mu_{r}-s-r+1} & H_{\mu_{r}-s-r+2} & \ldots & H_{\mu_{r}} \\
H_{-m-\nu_{s}} & H_{-m-\nu_{s}+1} & \ldots & H_{-\nu_{s}-1} \\
\vdots & \vdots & \ddots & \vdots \\
H_{-m-\nu_{1}-s+1} & H_{-m-\nu_{1}-s+2} & \ldots & H_{-\nu_{1}-s}
\end{array}\right|
$$

But it it easy to see from the definition, that

$$
\left(x_{1} \ldots x_{m}\right) E_{\lambda_{1} \ldots, \lambda_{m}}\left(x_{1}, \ldots, x_{m}\right)=E_{\lambda_{1}+1 \ldots, \lambda_{m}+1}\left(x_{1}, \ldots, x_{m}\right)
$$

and Lemma follows.

Now we are going to describe the ring $\Lambda_{m}^{ \pm}$in terms of generators and relations. First, we prove the following Lemma.

Lemma 2.10. Let $A$ be a matrix of the size $n \times(n+1)$ and

$$
A=\left(A_{1}, A_{2}, \ldots A_{n+1}\right)
$$

its columns. Let $A^{(l)}=A \backslash A_{l}$ be matrix of the size $n \times n$ obtaning from $A$ by deleting the column $A_{l}$. For any subset $I \subset\{1, \ldots, n\}$ define also matrix 
$A(I)$ of the size $n \times n$ by the formula

$$
r_{i}(A(I))=\left\{\begin{array}{l}
r_{i}\left(A^{(n+1)}\right), i \in I \\
r_{i}\left(A^{(1)}\right), i \notin I
\end{array}\right.
$$

where $r_{i}(B)$ denotes the $i$-th row of the matrix $B$.

Then the following equality is true

$$
\operatorname{det} A^{(l)}=\sum_{I \subset\{1, \ldots, n\}} \operatorname{det} A(I)
$$

and sum is taken over all subset cardinality $l-1$.

Proof. Let us consider matrix $A^{(1)}+a A^{(n+1)}$, where $a$ is indeterminate and decompose determinant in two ways: using rows and columns. If we use multilinear property of the determinant in rows, then we get

$$
\operatorname{det}\left(A^{(1)}+a A^{(n+1)}\right)=\sum_{l=0}^{n} a^{l} \sum_{\operatorname{Card}(I)=l} \operatorname{det} A_{I}
$$

where $\operatorname{Card}(I)$ denotes the number of elements in $I$. If we use multilinear property of the determinant in columns then we get

$$
\operatorname{det}\left(A^{(1)}+a A^{(n+1)}\right)=\sum_{l=1}^{n+1} a^{l-1} \operatorname{det} A^{(l)}
$$

and proof follows.

Definition 2.11. Let $z=\left(z_{i}\right), i \in \mathbb{Z}$ be an infinite sequence of variables and $I=\left(i_{1}, \ldots i_{p}\right) \in \mathbb{Z}^{p}$ finite sequence of integers. Set

$$
R_{I}(z)=\operatorname{det}\left(z_{i_{\alpha}+\beta-1}\right)_{1 \leq, \alpha, \beta \leq p}
$$

We will call $p$ as the length of $I$ and denote it by $\hat{l}(I)$.

Now we are ready to describe the ring $\Lambda_{m}^{ \pm}$in terms of the generators and relations.

Theorem 2.12. Ring $\Lambda_{m}^{ \pm}$is isomorphic any of the following rings:

1) $U_{m, 0}^{+}$which is generated by $u_{i}, \hat{v}_{i}, i \in \mathbb{N}$ with relations

$$
R_{I}(w)=0, \text { for any } I \in \mathbb{Z}^{m+1}
$$

where $w_{i}=u_{i}-\hat{v}_{-m-i}, i \in \mathbb{Z}$ and we suppose that $u_{0}=1 u u_{i}=\hat{v}_{i}=0, i<$ 0 .

2) $U_{m, 0}^{ \pm}$which is generated by $t, u_{i}, v_{i}, i \in \mathbb{N}$ with relations

$$
R_{I}(w)=0, \text { for any } I \in \mathbb{Z}^{m+1}
$$

where $w_{i}=u_{i}-t v_{-m-i}, i \in \mathbb{Z}$ and we assume that $u_{0}=v_{0}=1$ and $u_{i}=v_{i}=0, i<0$. 
Proof. Let us prove the first statement. Let $S=\left\{u_{1}, \ldots, \hat{v}_{0}, \ldots\right\}$ be the set of generators of the ring $U_{m, 0}$. Consider a map

$$
\varphi: S \longrightarrow \Lambda_{m}^{ \pm}, \varphi\left(u_{i}\right)=h_{i}, i \geq 1, \varphi\left(\hat{v}_{i}\right)=(-1)^{m}\left(x_{1} \ldots x_{m}\right)^{-1} h_{i}^{*}, i \geq 0
$$

Then it is easy to check that $\varphi\left(w_{i}\right)=H_{i}, i \in \mathbb{Z}$. Therefore the map $\varphi$ can be extended as the homomorphism on the whole ring $\varphi: U_{m, 0} \rightarrow \Lambda_{m}^{ \pm}$. Now let us prove that this map is an isomorphism. For that we are going to construct some family of linear generators of the ring $\Lambda_{m}^{ \pm}$. Namely we will prove that ring $U_{m, 0}$ is a linear span of the elements $R_{I}(w)$ where $I \in \mathbb{Z}^{m}$. It is enough to prove that any product $w_{j_{1}} \ldots w_{j_{r}}$ for any $r \geq 0$ can be expressed as a linear combination some of $R_{I}(w)$ with $\hat{l}(I)=m$. We will use induction on $r$. If $r=0$ then the product is equal to 1 and the following equality is easy to verify $1=R_{0,1, \ldots, 1-m}(w)$. So we can assume that $r>1$ and we will prove that product $R_{i_{1}, \ldots, i_{m}}(w) w_{j}$ where $j \notin\{0,-1, \ldots, 1-m\}$ is a linear combination some of $R_{j_{1}, \ldots, j_{m}}(w)$.

There are two cases $j>0$ and $j<1-m$. Consider the first case. We are going to use induction on $j$. If $j=1$, then by definition of the ring $U_{m, 0}$ we have $R_{\tilde{I}}(w)=0$, where $\tilde{I}=\left(i_{1}, i_{2}, \ldots, i_{m}, 1-m\right)$. Expanding the determinant along its last row we get

$$
\sum_{i \in\{1-m, \ldots, 0,1\}}(-1)^{i+1} R_{\tilde{I}}^{(i)}(w) w_{i}=0
$$

where $R_{\tilde{I}}^{(i)}(w)$ means the determinant obtaining from $R_{\tilde{I}}(w)$ by deleting row and column containing element $w_{i}$. Therefore

$$
R_{I}(w) w_{1}+\sum_{i \in\{1-m, \ldots, 0\}}(-1)^{i+1} R_{\tilde{I}}^{(i)}(w) w_{i}=0
$$

So $R_{I}(w) w_{1}=R_{\tilde{I}}^{(0)}(w)$. But if we apply Lemma 2.10 to the matrix

$$
A=\left(\begin{array}{cccc}
w_{i_{1}} & w_{i_{1}+1} & \ldots & w_{i_{1}+m} \\
\vdots & \vdots & \ddots & \vdots \\
w_{i_{m}} & w_{i_{l}+1} & \ldots & w_{i_{l}+m}
\end{array}\right)
$$

then we see that $R_{\tilde{I}}^{(0)}(w)$ can be expressed as a linear combination some of $R_{I}(w)$, with $\hat{l}(I)=m$. If $j>1$ then it is enough to consider relation $R_{\tilde{I}}(w)=0$, where $\tilde{I}=\left(i_{1}, \ldots, i_{m}, j-m\right)$ and to use inductive assumption. If $j<1-m$, then we can use the same arguments applying to relation $R_{\tilde{I}}(w)=0$, where $\tilde{I}=\left(i_{1}, \ldots, i_{m}, j\right)$. Thereby we proved that $U_{m, 0}$ is a linear span of $R\left(i_{1}, \ldots, i_{m}\right)$ with $\hat{l}(I)=m$.

Therefore by Theorem 2.6

$$
\varphi\left(R_{i_{1}, \ldots, i_{m}}(w)\right)=E_{i_{1}, i_{2}+1 \ldots, i_{m}+m-1}\left(x_{1}, \ldots, x_{m}\right)
$$


and by Theorem 2.3 elements $\varphi\left(R_{i_{1}, \ldots, i_{m}}(w)\right), i_{1}>i_{2}>\cdots>i_{m}$ form a basis of the ring $\Lambda_{m}^{ \pm}$. Therefore homomorphism $\varphi$ is an isomorphism and we have proved the first statement.

Now let us prove the second statement. We shall prove that $U_{m, 0}$ and $U_{m, 0}^{ \pm}$are isomorphic. From the definition of these rings there exists a homomorphism

$$
\varphi: U_{m, 0} \longrightarrow U_{m, 0}^{ \pm}, \quad \varphi\left(u_{i}\right)=u_{i}, i \geq 1, \varphi\left(\hat{v}_{i}\right)=t v_{i}, i \geq 0
$$

Let us construct an inverse homomorphism $\psi: U_{m, 0}^{ \pm} \rightarrow U_{m, 0}$. First let us prove that element $\hat{v}_{0}$ is invertible in the ring $U_{m, 0}$. Consider relation

$$
R_{0,1, \ldots, m}(w)=\left|\begin{array}{cccc}
w_{0} & w_{1} & \ldots & w_{m} \\
\vdots & \vdots & \ddots & \vdots \\
w_{-m} & w_{-m+1} & \ldots & w_{0}
\end{array}\right|=0
$$

Since $w_{0}=1, w_{-1}=\cdots=w_{1-m}=0$, we have an equality

$$
(-1)^{m} w_{0}\left|\begin{array}{cccc}
w_{1} & w_{2} & \ldots & w_{m} \\
\vdots & \vdots & \ddots & \vdots \\
w_{1-m} & w_{2-m} & \ldots & w_{1}
\end{array}\right|=-1
$$

Therefore $\hat{v}_{0}=-w_{m}$ is invertible and we can define homomorphism $\psi$

$$
\psi: U_{m, 0}^{ \pm} \longrightarrow U_{m, 0}, \quad \psi\left(u_{i}\right)=u_{i}, i \geq 1, \psi(t)=\hat{v}_{0}, \psi\left(v_{i}\right)=\hat{v}_{0}^{-1} \hat{v}_{i}, i \geq 1
$$

It is easy to check, that $\varphi, \psi$ are mutually inverse homomorphisms. This proves the second statement and the Theorem.

Remark 2.13. In the previous theorem we used two slightly different ways to define the ring of Laurent symmetric polynomials by means of generators and relations. We shall see later that a natural generalisation of the first way gives description of the ring of partially polynomial supersymmetric polynomials and a natural generalisation of the second way gives a description of the ring Laurent supersymmetric polynomials.

\section{Supersymmetric PARTIALly POLYNOMial AND POLYNOMIAL RINGS}

Definition 3.1. The following ring

$$
\Lambda_{m, n}^{+y}=\left\{f \in \mathbb{Z}\left[x_{1}^{ \pm 1}, \ldots, x_{m}^{ \pm 1}, y_{1}, \ldots, y_{n}\right] \mid x_{i} \frac{\partial f}{\partial x_{i}}+y_{j} \frac{\partial f}{\partial y_{j}} \in\left(x_{i}-y_{j}\right)\right\}
$$

will be called the ring of partially polynomial (in y-s) supersymmetric polynomials.

Definition 3.2. The ring

$$
\Lambda_{m, n}=\left\{f \in \mathbb{Z}\left[x_{1}, \ldots, x_{m}, y_{1}, \ldots, y_{n}\right] \mid x_{i} \frac{\partial f}{\partial x_{i}}+y_{j} \frac{\partial f}{\partial y_{j}} \in\left(x_{i}-y_{j}\right)\right\}
$$

will be called the ring of super symmetric polynomials. 
In this section we describe the rings $\Lambda_{m, n}^{+y}$ and $\Lambda_{m, n}$ in term of the generators and relations.

Let us define $h_{k}, h_{k}^{(\infty)}$ by means of expansion at zero and at infinity the following rational function

$$
\frac{\prod_{j=1}^{n}\left(1-y_{j} t\right)}{\prod_{i=1}^{m}\left(1-x_{i} t\right)}=\sum_{k=0}^{\infty} h_{k} t^{k}=\sum_{k=-\infty}^{n-m} h_{k}^{(\infty)} t^{k}
$$

It is easy to see that

$$
h_{k}^{(\infty)}=(-1)^{n-m} \frac{y_{1} \ldots y_{n}}{x_{1} \ldots x_{m}} h_{n-m-k}^{*},
$$

where $h_{k}^{*}=h_{k}\left(x_{1}^{-1}, \ldots, x_{m}^{-1}, y_{1}^{-1}, \ldots, y_{n}^{-1}\right)$. We also assume that $h_{k}^{*}=h_{k}=$ 0 , if $k<0$.

Definition 3.3. For $k \in \mathbb{Z}$ set

$$
H_{k}=h_{k}-h_{k}^{(\infty)}=h_{k}-(-1)^{n-m} \frac{y_{1} \ldots y_{n}}{x_{1} \ldots x_{m}} h_{n-m-k}^{*}
$$

Remark 3.4. Previous formulae can be rewritten in the form

$$
H_{k}=\left\{\begin{array}{l}
h_{k}, k>n-m \\
h_{k}-h_{k}^{(\infty)} \quad 0 \leq k \leq n-m \\
-h_{k}^{(\infty)} k<0
\end{array}\right.
$$

Lemma 3.5. The following equalities are valid

1)

$$
H_{k}(x, y)=\sum_{j=0}^{n}(-1)^{j} e_{j}(y) H_{k-j}(x)
$$

2)

$$
H_{k}(x) \Delta(x) \Delta(y)=\left\{\prod_{j=1}^{n}\left(1-\frac{y_{j}}{x_{1}}\right) x_{1}^{k} x_{1}^{m-1} x_{2}^{m-2} \ldots x_{m}^{0} y_{1}^{n-1} \ldots y_{n}^{0}\right\}
$$

and $\{f(x, y)\}$ means alternation over the group $S_{m} \times S_{n}$

$$
\{f(x, y)\}=\sum_{(\sigma, \tau) \in S_{m} \times S_{n}} \varepsilon(\sigma) \varepsilon(\tau) f(\sigma x, \tau y)
$$

3)

$$
H_{k}(x, y)-x_{1} H_{k-1}(x, y)=H_{k}\left(x_{2}, \ldots, x_{m}, y\right)
$$

4) if $m=1$, then for any integer $k$

$$
H_{k}\left(x_{1}, y\right)-x_{1} H_{k-1}\left(x_{1}, y\right)=0
$$

5) For any sequence of integers $\lambda_{1}, \ldots, \lambda_{m}$ the following equality is true

$$
\operatorname{det}\left(H_{\lambda_{i}-i+j}\right)_{1 \leq i, j \leq m}=\prod_{i=1}^{m} \prod_{j=1}^{n}\left(1-\frac{y_{j}}{x_{i}}\right) E_{\lambda}\left(x_{1}, \ldots, x_{m}\right)
$$


6) For any sequence of integers $\lambda_{1}, \ldots, \lambda_{m+1}$ we have the following equality

$$
\operatorname{det}\left(H_{\lambda_{i}-i+j}\right)_{1 \leq i, j \leq m+1}=0
$$

Proof. Let us prove the first statement. It is enough to prove it separately for $h_{k}$ и $h_{k}^{(\infty)}$. In the case of $h_{k}$ it follows from the equality

$$
\sum_{i=0}^{\infty} h_{k}(x, y) t^{k}=\left(\sum_{j=0}^{n}(-1)^{j} e_{j}(y) t^{j}\right) \sum_{i=0}^{\infty} h_{k}(x) t^{k}
$$

A proof for $h_{k}^{(\infty)}$ is similar.

The second statement follows from the first one and the definition of $H_{k}(x)$.

The third and the forth statements also follow from the first one and Lemma 2.5.

Statements 5),6) can be proved in the same manner as in Theorem 2.6. but instead of the definition $H_{k}(x)$ we need to use the statement 2) from Lemma 3.5 .

In order to describe the algebra $\Lambda_{m, n}^{+y}$ in terms of generators and relations we need to construct a linear basis in this algebra.

Definition 3.6. Let $I=\left(i_{1}, \ldots, i_{p}\right)$ be a sequence of integers and $J=$ $\left(j_{1}, \ldots, j_{q}\right)$ be a sequence of nonnegative integers. Set

$$
H(I, J)=\left|\begin{array}{cccc}
H_{i_{1}} & H_{i_{1}+1} & \ldots & H_{i_{1}+p-1} \\
\vdots & \vdots & \ddots & \vdots \\
H_{i_{p}} & H_{i_{p}} & \ldots & H_{i_{p}+p-1}
\end{array}\right| h_{1}^{j_{1}} h_{2}^{j_{2}} \ldots h_{q}^{j_{q}}=R_{I}(H) h^{J}
$$

Let us also denote by $X^{+}(m, n)$ the set of pairs of the sequences $(I, J)$ such that $I$ strictly decreasing sequence of integers, $J$ any sequence of nonnegative integers and

$$
\hat{l}(I) \leq m, \quad \hat{l}(J) \leq n, \quad \hat{l}(I)-\hat{l}(J)=m-n
$$

where as before the equality $\hat{l}(I)=p$ means that $I \in \mathbb{Z}^{p}$.

Remark 3.7. If $\hat{l}(I)=0$, then we assume that $I=\emptyset$ and $R_{I}(H)=1$ if $\hat{l}(J)=0$, then we assume that $h^{J}=1$.

Theorem 3.8. Elements $H(I, J),(I, J) \in X^{+}(m, n)$ form a linear basis of the ring $\Lambda_{m, n}^{+y}$.

Proof. Let use induction on $m n$. Let $m n=0$. If $n=0$, then the statement follows from Theorem 2.6. If $m=0$, the the statement follows from the main theorem of symmetric functions. Let $m n>0$. L Consider a natural homomorphism

$$
\varphi_{m, n}: \Lambda_{m, n}^{+y} \longrightarrow \Lambda_{m-1, n-1}^{+y}
$$


such that $\varphi_{m, n}\left(x_{m}\right)=\varphi_{n, m}\left(y_{n}\right)=t$, and it acts identically on all other variables. It is clear that

$$
\varphi\left(H_{i}\right)=H_{i}, \varphi\left(h_{j}\right)=h_{j}
$$

From the inductive assumption it follows that this homomorphism is surjection. Therefore it is enough to prove that the kernel of this homomorphism has a basis consisting of $H(I, J)$ such, that $\hat{l}(I)=m, \hat{l}(J)=n$. It is easy to check that the following family forms a basis of the kernel

$$
\prod_{i=1}^{m} \prod_{j=1}^{n}\left(1-\frac{y_{j}}{x_{i}}\right) E_{\lambda}(x) e_{1}(y)^{j_{1}} \ldots e_{n}(y)^{j_{n}}
$$

where $\lambda_{1} \geq \lambda_{2} \geq \cdots \geq \lambda_{m}$ non-increasing sequence of integers and $j_{1}, \ldots, j_{n}$ - any sequence of nonnegative integers and $e_{1}, \ldots, e_{n}$ elementary symmetric polynomials. Further we have

$$
\begin{gathered}
h_{1}(x, y)=h_{1}(x)-e_{1}(y) \\
h_{2}(x, y)=h_{2}(x)-h_{1}(x) e_{1}(y)+e_{2}(y) \\
\vdots \\
h_{n}(x, y)=h_{n}(x)-h_{n-1}(x) e_{1}(y)+\cdots+(-1)^{n} e_{n}(y)
\end{gathered}
$$

So we see that $h_{1}(x, y), h_{2}(x, y), \ldots, h_{n}(x, y)$ can be expressed by low-triangular matrix in terms of $e_{1}(y), \sigma_{2}(y), \ldots, e_{n}(y)$ with units (up to sign) on the main diagonal. Therefore there exists an automorphism $\sigma$ of the algebra $\mathbb{C}\left[x_{1}^{ \pm 1}, \ldots, x_{m}^{ \pm 1}, y_{1}, \ldots, y_{n}\right]^{S_{m} \times S_{m}}$ such that

$$
\sigma\left(e_{i}(y)\right)=h_{i}(x, y), i=1, \ldots, n, \quad \sigma\left(e_{i}(x)\right)=e_{i}(x), i=1, \ldots, m .
$$

Therefore by Lemma 3.5 we see that

$$
R_{\lambda_{1}, \lambda_{2}+1, \ldots, \lambda_{m}+m-1} h_{1}^{j_{1}} h_{2}^{j_{2}} \ldots h_{n}^{j_{n}}
$$

also form a basis of the kernel. And the Theorem follows from the inductive assumption.

Definition 3.9. Let $m, n$ be two nonnegative integers. Let also $u_{1}, u_{2}, \ldots$ and $v_{0}, v_{1}, \ldots$ be two infinite sets. We will assume that $u_{0}=1$ and $u_{i}=$ $0, v_{i}=0$ for $i<0$. Set $w_{i}=u_{i}-v_{-i-m+n}, i \in \mathbb{Z}$ and denote by $U_{m, n}^{+}$the ring generated by $u_{1}, u_{2}, \ldots$, and $v_{0}, v_{1}, \ldots$, with relations

$$
R_{i_{1}, \ldots, i_{m+1}}(w)=0, \text { for any }\left(i_{1}, \ldots, i_{m+1}\right) \in \mathbb{Z}^{m+1}
$$

Now we want to construct some set of linear generator of the algebra $U_{m, n}$.

Definition 3.10. Let $I=\left(i_{1}, \ldots, i_{p}\right)$ be a sequence of integers and $J=$ $\left(j_{1}, \ldots, j_{q}\right)$ be a sequence of nonnegative integers. Set

$$
R(I, J)=R_{I}(w) u_{1}^{j_{1}} u_{2}^{j_{2}} \ldots u_{q}^{j_{q}}
$$

Theorem 3.11. Elements $R(I, J)$, such that $(I, J) \in X^{+}(m, n)$ linearly generate the ring $U_{m, n}^{+}$. 
Proof. We will use induction on $m n$. Let $m n=0$. Then, either $m=0$, or $n=0$. In the first case $\hat{l}(I)=0, \hat{l}(J)=n$, Therefore the relations become $R_{i}(w)=0, i \in \mathbb{Z}$ and they are equivalent to the relations $u_{i}=0, i>n$ and $u_{i}-v_{n-i}=0,0 \leq i \leq n$ and $v_{j}=0, j>n$. Therefore $U^{+}(0, n)$ is generated algebraically by $u_{1}, \ldots, u_{n}$ and in this case Theorem is true.

In the second case $\hat{l}(I)=m, \hat{l}(J)=0$ and we need to show that $U^{+}(m, 0)$ is a linear span of the elements $R_{I}(w), \hat{l}(I)=m$. But this follows from the Theorem 2.12.

Let us now suppose that $m n>0$. We have $m-n=(m-1)-(n-1)$, therefore from the definition of the rings $U_{m, n}^{+}$it follows that there exists a homomorphism

$$
\psi_{m, n}: U_{n, m}^{+} \rightarrow U_{m-1, n-1}^{+}
$$

which sends generators to generators. Again from the definition of the rings $U_{n, m}^{+}$it follows that the kernel of this homomorphism is the ideal generated by $R_{I}(w)$ with $\hat{l}(I)=m$. Therefore it is enough to prove that for $j \neq$ $1, \ldots, n$ the product $R_{I}(w) u_{j}$ can be expressed as a linear combination some of $R(\tilde{I}, \tilde{J})$. And we need to prove also that product $R_{I} v_{j}$ for any $j$ is a linear combination some of $R(\tilde{I}, \tilde{J})$ as well.

Let us consider the first case. We can assume that $j \geq n$ and we will use induction on $j-n$. If $j=n$, then our statement is clear. Let $j>n$. Consider relation $R_{i_{1}, \ldots, i_{m}, j-m}(w)=0$. If we expand the determinant along the last row then we get

$$
R_{I}(w) w_{j}+\sum_{i \in\{j-m, \ldots, j-1\}}(-1)^{i+1} R_{\tilde{I}}^{(i)}(w) w_{i}=0
$$

Since $i \in\{j-m, \ldots, j-1\}$ we have $i \geq j-m>n-m$, so $w_{i}=u_{i}$ (it would be zero, if $i<0)$ and by induction $R_{I}(w) w_{j}$ is a linear combination some of $R(I, J)$. Let us prove now that $R_{I} v_{j}$ with $\hat{l}(I)=m$ is a linear combination some of $R(\tilde{I}, \tilde{J})$ for $j \leq 0$ using induction on $j$. If $j=0$, then $w_{n-m}=u_{n-m}-v_{0}$. Therefore we can replace $v_{0}$ on $w_{n-m}$ and we can consider relation $R_{i_{1}, \ldots, i_{m}, n-m}=0$. Using the same arguments as before we get necessary statement for $j=0$. If $j>0$, then $w_{n-m-j}=u_{n-m-j}-v_{j}$ and we can replace $v_{j}$ by $w_{n-m-j}$ and we can consider a relation $R_{i_{1}, \ldots, i_{m}, n-m-j}=$ 0 and use inductive assumption. Theorem is proved.

Corollary 3.12. Rings $\Lambda_{m, n}^{+y}$ and $U_{m, n}^{+}$are isomorphic.

Proof. By Lemma 3.5

$$
\operatorname{det}\left(H_{\lambda_{i}-i+j}\right)_{1 \leq i, j \leq m+1}=0
$$

for any sequence of integers $\lambda_{1}, \ldots, \lambda_{m+1}$. Therefore from the defining relations of the ring $U_{m, n}^{+}$it follows that there exists homomorphism such, that

$$
\varphi: U_{m, n} \longrightarrow \Lambda_{m, n}^{+y}, \varphi\left(u_{i}\right)=h_{i}, \varphi\left(v_{i}\right)=(-1)^{n-m} \frac{y_{1} \ldots y_{n}}{x_{1} \ldots x_{m}} h_{i}^{*}, i \geq 1
$$


This homomorphism sends the family of linear generators of the algebra $U_{m, n}^{+}$ to a basis of the algebra $\Lambda_{m, n}^{+y}$. Therefore it is an isomorphism.

Let us consider the ring $\Lambda_{m, n}$ of supersymmetric polynomials. It is a subring in $\Lambda_{m, n}^{+y}$. We also want to describe it in terms of generators and relations. Let us denote by $\mathbb{Z}_{>a}$ the set of integers which are strictly grater then $a$.

Corollary 3.13. $\Lambda_{m, n}$ is isomorphic to the ring $U_{m, n}$ which is generated by $u_{1}, u_{2}, \ldots$ subject to relations

$$
R_{I}(u)=0, \text { for any } I=\left(i_{1}, \ldots, i_{m+1}\right) \in\left(\mathbb{Z}_{>n-m}\right)^{m+1}
$$

and we assume that $u_{0}=1 u u_{i}=0, i<0$.

Proof. It is well known that ring $\Lambda_{m, n}$ is generated by $h_{1}, h_{2}, \ldots$ ( see for example [9]). Therefore according to the previous Theorem the ring $\Lambda_{m, n}$ is isomorphic to the subring $U$ in $U_{m, n}$ generated by $u_{1}, u_{2}, \ldots$ From the definition $w_{i}$ it follows that if $i>n-m$ then $w_{i}=u_{i}$. Therefore elements $R(I, J), l(I)=m, I \in\left(\mathbb{Z}_{>n-m}\right)^{m}$ (we call such elements admissible) belong to $U$.

Let us consider relation $R_{I}(u)=0, \hat{l}(I)=m+1$, where $I$ is admissible (we call such relations also admissible). Then by the same arguments as in the proof of the Theorem 4.7. it can be shown that admissible elements linearly generate $U$ by using only admissible relations. But according to the corollary 3.12 admissible elements $R(I, J)$ are linearly independent. This proves corollary.

Corollary 3.14. Let $n \geq m$, then the ring $\Lambda_{m, n}^{+y}$ (as the graded one) is isomorphic to the ring $\Lambda_{m, m}^{+y} \otimes \Lambda_{n-m}$.

Proof. Let us consider a map $\varphi: \Lambda_{m, m}^{+} \longrightarrow \Lambda_{m, n}^{+}$,

$$
\varphi\left(h_{i}\right)=h_{n-m+i}, \quad \varphi\left(\frac{y_{1} \ldots y_{m}}{x_{1} \ldots x_{m}} h_{i}^{*}\right)=(-1)^{n-m} \frac{y_{1} \ldots y_{n}}{x_{1} \ldots x_{m}} h_{i}^{*}, i \geq 1
$$

It follows from the defining relations of the ring $\Lambda_{m, m}^{+}$that the map $\varphi$ can be extended to the homomorphism of the rings $\varphi: \Lambda_{m, m} \longrightarrow \Lambda_{m, n}$ It is easy to check that $\varphi\left(H_{i}\right)=H_{n-m+i}$, and $\varphi(H(I, J))=H(I+n-m, J+n-m)$, where $I+a$ means the sequence $\left(i_{1}+a, \ldots, i_{p}+a\right)$. So the map $\varphi$ sends the basis of the ring $\Lambda_{m, m}^{+}$to a subset of the basis of the ring $\Lambda_{m, n}$. And $\varphi$ is injective when restricted to the basis. Therefore $\varphi$ is injective as a homomorphism of the rings.

Further there exists a homomorphism $\psi$ such that

$$
\psi: \Lambda_{n-m} \longrightarrow \Lambda_{m, n}, \psi\left(h_{i}\right)=h_{i}, i=1, \ldots, n-m
$$

So we have a homomorphism

$$
\varphi \otimes \psi: \Lambda_{m, m}^{+y} \otimes \Lambda_{m, n} \longrightarrow \Lambda_{m, n}
$$

and it is easy to see that homomorphism $\varphi \otimes \psi$ sends bijectively tensor product of the bases in rings $\Lambda_{m, m}$ and $\Lambda_{n-m}$ to the basis of the ring $\Lambda_{m, n}$. 
Therefore this is an isomorphism. But it does not preserves the grading. In order to construct a homomorphism preserving the grading let us consider a composition $\varphi \otimes \psi \circ \delta$, where $\delta: \Lambda_{m, m}^{+y} \longrightarrow \Lambda_{m, m}^{+y}$ is an automorphism $\delta\left(H_{i}\right)=H_{i-n+m}$.

Corollary 3.15. Let $n \geq m$. Then there exists an isomorphism of rings

$$
\Lambda_{m, m} \otimes \Lambda_{n-m}=\Lambda_{m, n}
$$

Proof. Let us consider the previous isomorphism $\varphi \otimes \psi$ and restrict it to the subring $\Lambda_{m, m} \otimes \Lambda_{n-m}$. It is clear that its image is $\Lambda_{m, n}$.

Remark 3.16. It it easy to see that the isomorphism from the previous corollary does not preserves the grading.

\section{LAURENT SUPERSYMMETRIC POLYNOMIALS}

Definition 4.1. The following ring

$$
\Lambda_{m, n}^{ \pm}=\left\{f \in \mathbb{Z}\left[x_{1}^{ \pm 1}, \ldots, x_{m}^{ \pm 1}, y_{1}^{ \pm 1}, \ldots, y_{n}^{ \pm 1}\right] \mid x_{i} \frac{\partial f}{\partial x_{i}}+y_{j} \frac{\partial f}{\partial y_{j}} \in\left(x_{i}-y_{j}\right)\right\}
$$

will be called the ring of Laurent supersymmetric polynomials.

We are going to describe it in term of generators and relations. First we are going to construct some natural basis in this ring.

Definition 4.2. Let $I=\left(i_{1}, \ldots, i_{p}\right)$ be a sequence of integers and $J=$ $\left(j_{1}, \ldots, j_{q}\right)$ be a sequence of integers such that the first $q-1$ elements are nonnegative and the last one is any integer. Let us set

$$
H(I, J)=R_{I}(H) h_{1}^{j_{1}} h_{2}^{j_{2}} \ldots h_{q-1}^{j_{q-1}} \Delta^{j_{q}}
$$

where $\Delta=\frac{y_{1} \ldots y_{n}}{x_{1} \ldots x_{m}}$.

Let us denote by $X^{ \pm}(m, n)$ the set of pairs $(I, J)$ with the same properties as in Definition 4.2. but sequence $I$ is strictly decreasing and such that

$$
\hat{l}(I) \leq m, \hat{l}(J) \leq n, \hat{l}(I)-\hat{l}(J)=m-n
$$

Theorem 4.3. Let $(I, J) \in X^{ \pm}(m, n)$, then elements $H(I, J)$ form a linear basis of the ring $\Lambda_{m, n}^{ \pm}$.

Proof. Let us use induction on $m n$. Let $m n=0$. If $n=0$, then $\hat{l}(I)=m$ and the statement follows from Theorem 2.6. If $m=0$, the statement follows form the fact that the set of element of the type $e_{1}^{j_{1}} \ldots e_{n-1}^{j_{n-1}} e_{n}^{j_{n}}$ form a linear basis in the ring of Laurent symmetric polynomials $\mathbb{Z}\left[y_{1}^{ \pm 1}, \ldots, y_{n}^{ \pm 1}\right]^{S_{n}}$.

Let $m n>0$. Consider a natural homomorphism

$$
\varphi_{m, n}: \Lambda_{m, n}^{ \pm} \longrightarrow \Lambda_{m-1, n-1}^{ \pm}
$$


such that $\varphi_{m, n}\left(x_{m}\right)=\varphi_{n, m}\left(y_{n}\right)=t$, and it acts identically on all other variables. It is clear that

$$
\varphi\left(H_{i}\right)=H_{i}, i \in \mathbb{Z}, \quad \varphi\left(h_{j}\right)=h_{j}, \quad \varphi\left(h_{j}^{*}\right)=h_{j}^{*}, j \in \mathbb{Z}_{\geq 0} \quad \varphi(\Delta)=\Delta
$$

From the inductive assumption it follows that this homomorphism is surjection and it is enough to prove that the kernel of this homomorphism has a basis consisting of $H(I, J)$ such that $\hat{l}(I)=m, \hat{l}(J)=n$. It is easy to check that that the following family of elements forms a basis of the kernel

$$
\prod_{i=1}^{m} \prod_{j=1}^{n}\left(1-\frac{y_{j}}{x_{i}}\right) E_{\lambda}(x) e_{1}(y)^{j_{1}} \ldots e_{n}(y)^{j_{n}}
$$

where $\lambda_{1} \geq \lambda_{2} \geq \cdots \geq \lambda_{m}$ is a non-increasing sequence of integers and $j_{1}, \ldots, j_{n-1}$ is any sequence of nonnegative integers, $j_{n}$ is any integer and $e_{1}, \ldots, e_{n}$ elementary symmetric polynomials. Further we have

$$
\begin{gathered}
h_{1}(x, y)=h_{1}(x)-e_{1}(y) \\
h_{2}(x, y)=h_{2}(x)-h_{1}(x) e_{1}(y)+e_{2}(y) \\
\vdots \quad \vdots \quad \vdots \quad \vdots \\
h_{n-1}(x, y)=h_{n-1}(x)-h_{n-2}(x) e_{1}(y)+\cdots+(-1)^{n} e_{n-1}(y) \\
\Delta=\frac{e_{n}(y)}{e_{m}(x)}
\end{gathered}
$$

So we see that $h_{1}(x, y), h_{2}(x, y), \ldots, h_{n-1}(x, y), \Delta$ can be expressed by lowtriangular matrix in terms of $e_{1}(y), e_{2}(y), \ldots, e_{n}(y)$ with units and $e_{m}(x)^{-1}$ (up to sign) on the main diagonal. Therefore there exists an automorphism $\sigma$ of the ring

$$
\mathbb{Z}\left[x_{1}^{ \pm 1}, \ldots, x_{m}^{ \pm 1}, y_{1}^{ \pm 1}, \ldots, y_{n}^{ \pm 1}\right]^{S_{m} \times S_{m}}
$$

such that

$$
\begin{gathered}
\varphi\left(\sigma_{i}(y)\right)=h_{i}(x, y), i=1, \ldots, n-1, \varphi\left(\sigma_{n}(y)\right)=\Delta, \\
\varphi\left(\sigma_{i}(x)\right)=\sigma_{i}(x), i=1, \ldots, m .
\end{gathered}
$$

Therefore by Lemma 3.5 we see that

$$
H_{\lambda_{1}, \ldots, \lambda_{m}} h_{1}^{j_{1}} h_{2}^{j_{2}} \ldots h_{n-1}^{j_{n-1}} \Delta^{j_{n}}
$$

also form a basis of the kernel. And the Theorem follows from the inductive assumption.

Definition 4.4. Let $m, n$ be two nonnegative integers. Let also $u_{1}, u_{2}, \ldots$, $v_{1}, v_{2}, \ldots$ be two infinite sets of variables and $t$ is an additional variable. We assume that $u_{0}=1, v_{0}=1$ and $u_{i}=0, v_{i}=0$ for $i<0$. Set $w_{i}=$ $u_{i}-t v_{-i-m+n}, i \in \mathbb{Z}$ and denote by $U_{m, n}^{ \pm}$the ring generated by $u_{1}, u_{2}, \ldots$, $v_{1}, v_{2}, \ldots, t$, and relations

$$
R_{i_{1}, \ldots, i_{m+1}}(w)=0, \text { for any }\left(i_{1}, \ldots, i_{m+1}\right) \in \mathbb{Z}^{m+1}
$$


We want to show that $\Lambda_{m, n}^{ \pm}$is isomorphic to $U_{m, n}^{ \pm}$. Let us prove first that $t$ is invertible in the ring $U_{m, n}^{ \pm}$.

Lemma 4.5. Element $t$ is invertible in the ring $U_{m, n}^{ \pm}$.

Proof. Let us consider the subring $\mathfrak{A} \subset U_{m, n}^{ \pm}$generated by $u_{i}, v_{i}, i \geq 1$. Let us prove that element $t$ satisfies algebraic equation with coefficients in $\mathfrak{A}$ and that the constant term is equal to 1 .

For that consider a relation

$$
\left|\begin{array}{cccc}
w_{0} & w_{1} & \ldots & w_{m} \\
\vdots & \vdots & \ddots & \vdots \\
w_{-m} & w_{-m+1} & \ldots & w_{0}
\end{array}\right|=0
$$

The left hand side of this relation is a polynomial in $t$. If we substitute in this relation $t=0$ we see that the constant term is 1 . Therefore the equation has a form

$$
a_{k} t^{k}+a_{k-1} t^{k-1}+\cdots+a_{1} t+1=0
$$

or

$$
t\left(a_{k} t^{k-1}+a_{k-1} t^{k-2}+\cdots+a_{1}\right)+1=0
$$

therefore $t$ is invertible.

Now we want to construct some set of linear generators of the ring $U_{m, n}^{ \pm}$.

Definition 4.6. Let $I=\left(i_{1}, \ldots, i_{p}\right), J=\left(j_{1}, \ldots, j_{q}\right)$ such that $(I, J) \in$ $X^{ \pm}(m, n)$. Set

$$
R(I, J)=R_{I}(w) u_{1}^{j_{1}} u_{2}^{j_{2}} \ldots u_{q-1}^{j_{q-1}} t^{j_{q}}
$$

Theorem 4.7. Let $(I, J) \in X^{ \pm}(m, n)$ then elements $R(I, J)$ linearly generate the ring $U_{m, n}^{ \pm}$.

Proof. Since determinant changes its sign after transposition of two rows we can suppose that members of the sequence $I$ does not necessary strictly decrease. We will use induction on $m n$. Let $m n=0$. Then either $m=0$, or $n=0$. In the first case $\hat{l}(I)=0, \hat{l}(J)=n$. Therefore the relations come to the form $w_{i}=0, i \in \mathbb{Z}$ and they are equivalent to the relations $u_{i}=0, v_{i}=0$, if $i>n$ and $1-t v_{n}=0, u_{n}-t=0, u_{i}-t v_{n-i}=0,1 \leq i \leq n-1$. Therefore the ring $U^{ \pm}(0, n)$ is generated algebraically by $u_{1}, \ldots, u_{n-1}, t, t^{-1}$ and Theorem is true in this case.

In the second case $\hat{l}(I)=m, \hat{l}(J)=0$ and we need to show that $U^{ \pm}(m, 0)$ is a linear span of the elements $R_{I}, \hat{l}(I)=m$. But this follows from the Theorem 2.12.

Suppose now that $m n>0$. We have $m-n=(m-1)-(n-1)$, therefore from the defining relations of the ring $U_{m, n}^{ \pm}$it follows that there exists a homomorphism

$$
\psi: U_{n, m}^{ \pm} \rightarrow U_{m-1, n-1}^{ \pm}
$$

which sends generators to generators. Again from the definition of the algebra $U_{n, m}^{ \pm}$it follows that the kernel of this homomorphism is the ideal 
generated by $R_{I}(w), l(I)=m$. Therefore in order to prove the Theorem it is enough to prove that product $R_{I}(w) u_{j}$ for $j \neq 1, \ldots, n-1$ can be expressed as a linear combination some of $R(\tilde{I}, \tilde{J}),(\tilde{I}, \tilde{J}) \in X^{ \pm}(m, n)$. And we also need to prove the same for $R_{I}(w) v_{j}$, for any $j$.

Let us consider the first case. We will prove this statement induction on $j-n+1$. If $j=n-1$, then it is clear. Let $j>n-1$. Consider relation $R i_{1}, \ldots, i_{m}, j-m(w)=0$. If we expand the determinant along its last row we get

$$
R_{I}(w) w_{j}+\sum_{i \in\{j-m, \ldots, j-1\}}(-1)^{i+1} R_{\tilde{I}}^{(i)}(w) w_{i}=0
$$

Since for $i \in\{j-m, \ldots, j-1\}$ we have $i \geq j-m \geq n-m$, then $w_{i}=u_{i}, u_{i}-t$ (the last case is possible if $j=n$ ) and by induction and Lemma 2.10 $R_{I}(w) w_{j}$ is a linear combination some of $R(I, J)$.

Let us prove the same statement for product $R_{I}(w) v_{j}$ and $j>0$ also by induction. If $j=1$, then $w_{n-m-1}=u_{n-m-1}-t v_{1}$ therefore we can replace $v_{1}$ by $w_{n-m-1}$. Then we can consider relation $R_{i_{1}, \ldots, i_{m}, n-m-1}(w)=0$. Using the same argument as before we get the necessary statement for $j=1$. Let $j>1$, then $w_{n-m-j}=u_{n-m-j}-t v_{j}$ and we can replace $v_{j}$ на $w_{n-m-j}$ and can consider relation $R_{i_{1}, \ldots, i_{m}, n-m-j}(w)=0$. Using this relation and inductive assumption we prove the Theorem.

Corollary 4.8. Rings $\Lambda_{m, n}^{ \pm}$and $U_{m, n}^{ \pm}$are isomorphic.

Proof. Let us consider a map $\varphi$

$$
\varphi\left(u_{i}\right)=h_{i}, \varphi\left(v_{i}\right)=h_{i}^{*}, i \geq 1, \varphi(t)=(-1)^{n-m} \frac{y_{1} \ldots y_{n}}{x_{1} \ldots x_{m}}
$$

By Lemma 3.5

$$
\operatorname{det}\left(H_{\lambda_{i}-i+j}\right)_{1 \leq i, j \leq m+1}=0
$$

Therefore the map $\varphi$ can be extended to the homomorphism

$$
\varphi: U_{m, n} \longrightarrow \Lambda_{m, n}^{+y} \text {. }
$$

It is easy to check that homomorphism $\varphi$ sends the family of linear generators of the ring $U_{m, n}$ to the basis of the ring $\Lambda_{m, n}^{+y}$. Therefore this is an isomorphism.

\section{JACOBI-TRudy FORMulae AND Euler Supercharacters}

It is well known that Schur polynomials is a natural basis of the ring $\Lambda_{m}$ and super Schur polynomials is a natural basis of the ring $\Lambda_{m, n}$. In the case of the rings $\Lambda_{m, n}^{+y}$ and $\Lambda_{m, n}^{ \pm}$there is a natural basis consisting of the supercharacters of irreducible finite dimensional modules as well. But until now a closed explicit formula for them is not known. It is possible to try to use super-analogues of composite Schur functions (see [11]), but they do not generate the whole algebra $\Lambda_{m, n}^{ \pm}$in general. So we use Euler supercharacters 
instead. There is an explicit formula for them according to Serganova [5] and they linearly generate the algebra $\Lambda_{m, n}^{ \pm}$. Of course there are many families of Euler supercharacters which form a basis in the algebra $\Lambda_{m, n}^{ \pm}$. We chose those which are closely related to the Kac modules and they are a natural generalisation of super Schur polynomials from the Jacobi-Trudy formula point of view.

First, we prove some technical lemmas. Let $a_{i}, b_{i}, i \geq 0$ be two sequences of elements from a commutative algebra $\mathfrak{A}$ such that $a_{0}=b_{0}=1$. Consider two formal series $f(t)=\sum_{i \geq 0} a_{i} t^{i}, \quad g(t)=\sum_{i \geq 0} b_{i} t^{i}$ and suppose that $f(t) g(t)=1$.

Remark 5.1. For a partition $\lambda$ we will denote as usual by $l(\lambda)$ the length of $\lambda$ and by $|\lambda|$ the number $\lambda_{1}+\lambda_{2}+\ldots$. We can also consider a partition $\lambda$ as a sequence of nonnegative integers $\lambda=\left(\lambda_{1}, \lambda_{2}, \ldots,\right)$. In this case the number $\hat{l}(\lambda)$ is also defined. But this number is always grater or equal to $l(\lambda)$ and it depends on how many zeros we put at the end of the $\lambda$. For example, let $\lambda=(3,3,2,2,1,0,0,0,0)$. Then $l(\lambda)=5$ but $\hat{l}(\lambda)=9$.

Lemma 5.2. Let $\lambda$ be a partition such that $l(\lambda) \leq p, l\left(\lambda^{\prime}\right) \leq r$. Then

$$
\operatorname{det}\left(a_{\lambda_{i}-i+j}\right)_{1 \leq i, j \leq p}=(-1)^{|\lambda|} \operatorname{det}\left(b_{\lambda_{i}^{\prime}-i+j}\right)_{1 \leq i, j \leq r}
$$

Proof. See [9]. For reader convenience we reproduce the proof here. Let us define two matrices $A=\left(a_{i-j}\right)$ and $B=\left(b_{i-j}\right)$ (we assume that $a_{i}=b_{i}=0$ for $i<0)$. Then the previous condition means that $A B=1$ and $\operatorname{det} A=$ $\operatorname{det} B=1$. By the formula for minors of mutually inverse matrices (see [4]) we have

$$
A(I, J)=(-1)^{|I|+|J|} B(\bar{J}, \bar{I}), \quad I, J \subset\{1, \ldots, N\},
$$

and $\bar{I}, \bar{J}$ are complements to $I, J$. Set $J=\{1, \ldots, p\}, I=\left\{\lambda_{p}+1, \lambda_{p-1}+\right.$ $\left.2, \ldots, \lambda_{1}+p\right\}$. Then $\bar{J}=\{p+1, \ldots, p+r\}$ and according to $[9] \bar{I}=\left\{p+j-\lambda_{j}^{\prime}\right\}$. Therefore

$$
\begin{gathered}
A(I, J)=\operatorname{det}\left(a_{i j}\right)_{i \in I, j \in J}=\operatorname{det}\left(a_{p-i+1, p-j+1}\right)=\operatorname{det}\left(a_{\lambda_{i}-i+j}\right) \\
B(\bar{J} \bar{I})=\operatorname{det}\left(b_{j i}\right)_{j \in \bar{J}, i \in \bar{I}}=\operatorname{det}\left(b_{p+j-p-i+\lambda_{i}^{\prime}}\right)=\operatorname{det}\left(b_{\lambda_{i}^{\prime}-i+j}\right)
\end{gathered}
$$

We also need a dual form of composite symmetric polynomials.

Lemma 5.3. Let $\nu$ and $\mu$ be partitions such $l(\mu)+l(\nu) \leq m$. Then the following equality is valid in the ring $\Lambda_{m}^{ \pm}$

$$
\left|\begin{array}{cccc}
h_{\nu_{q}}^{*} & h_{\nu_{q}-1}^{*} & \ldots & h_{\nu_{q}-q-p+1}^{*} \\
\vdots & \vdots & \ddots & \vdots \\
h_{\nu_{1}+q-1}^{*} & h_{\nu_{1}+q-2}^{*} & \ldots & h_{\nu_{1}-p}^{*} \\
h_{\mu_{1}-q} & h_{\mu_{1}-q+1} & \ldots & h_{\mu_{1}+p-1} \\
\vdots & \vdots & \ddots & \vdots \\
h_{\mu_{p}-q-p+1} & h_{\mu_{p}-q-p+2} & \ldots & h_{\mu_{p}}
\end{array}\right|=
$$




$$
\left|\begin{array}{cccc}
e_{\nu_{s}^{\prime}}^{*} & e_{\nu_{s}^{\prime}-1}^{*} & \ldots & e_{\nu_{s}^{\prime}-s-r+1}^{*} \\
\vdots & \vdots & \ddots & \vdots \\
e_{\nu_{1}^{\prime}+s-1}^{*} & e_{\nu_{1}^{\prime}+s-2}^{*} & \ldots & e_{\nu_{1}^{\prime}-r}^{*} \\
e_{\mu_{1}^{\prime}-s} & e_{\mu_{1}^{\prime}-s+1} & \ldots & e_{\mu_{1}^{\prime}+r-1} \\
\vdots & \vdots & \ddots & \vdots \\
e_{\mu_{r}^{\prime}-s-r+1} & e_{\mu_{r}^{\prime}-s-r+2} & \ldots & e_{\mu_{r}^{\prime}}
\end{array}\right|
$$

Proof. For any $i$ we have $e_{i}^{*}=e_{m}^{*} e_{m-i}$. Therefore the determinant on the right hand side takes a form

$$
\left(e_{m}^{*}\right)^{s}\left|\begin{array}{cccc}
e_{m-\nu_{s}^{\prime}} & e_{m-\nu_{s}^{\prime}+1} & \ldots & e_{m-\nu_{s}^{\prime}+s+r-1} \\
\vdots & \vdots & \ddots & \vdots \\
e_{m-\nu_{1}^{\prime}-s+1} & e_{m-\nu_{1}^{\prime}-s+2} & \ldots & e_{m-\nu_{1}^{\prime}+r} \\
e_{\mu_{1}^{\prime}-s} & e_{\mu_{1}^{\prime}-s+1} & \ldots & e_{\mu_{1}^{\prime}+r-1} \\
\vdots & \vdots & \ddots & \vdots \\
e_{\mu_{r}^{\prime}-s-r+1} & e_{\mu_{r}^{\prime}-s-r+2} & \ldots & e_{\mu_{r}^{\prime}}
\end{array}\right|
$$

According to our assumptions $m-\nu_{1}^{\prime} \geq \mu_{1}^{\prime}$, so by Lemma 5.2 the previous determinant is equal to

$$
\left(e_{m}^{*}\right)^{s}\left|\begin{array}{cccc}
h_{\lambda_{1}} & h_{\lambda_{1}+1} & \ldots & h_{\lambda_{1}+q+p-1} \\
\vdots & \vdots & \ddots & \vdots \\
h_{\lambda_{p+q}-q-p+1} & h_{\mu_{p+q}-q-p+2} & \ldots & h_{\lambda_{p+q}}
\end{array}\right|
$$

where $\lambda$ is the partition conjugated to partition $\left(m-\nu_{s}^{\prime}, \ldots, m-\nu_{1}^{\prime}, \mu_{1}^{\prime}, \ldots, \mu_{r}^{\prime}\right)$. It is easy to check that $\lambda=\left(\mu_{1}+\nu_{1}, \ldots, \mu_{p}+\nu_{1}, \nu_{1}-\nu_{q}, \ldots, \nu_{1}-\nu_{2}, 0\right)$. Therefore by Lemma 2.9 the right hand side of the equality which we are proving is equal to $E_{\chi}$, where $\chi=\left(\mu_{1}, \ldots, \mu_{p}, 0, \ldots, 0,-\nu_{s}, \ldots,-\nu_{1}\right)$. The left hand side is also equal to $E_{\chi}$ by the same Lemma.

We actually need a generalisation of the Lemma 5.2. There should be a direct proof the Lemma below. But we will use the previous Lemma instead. Let $a_{i}, a_{i}^{*}, b_{i}, b_{i}^{*}$ be four sequences of elements from a commutative algebra $\mathfrak{A}$ such that $a_{0}=a_{0}^{*}=b_{0}=b_{0}^{*}=1$ and $a_{i},=a_{i}^{*}=b_{i}=b_{i}^{*}=0$ for $i<0$. Consider four formal series

$$
f(t)=\sum_{i \geq 0} a_{i} t^{i}, \quad f^{*}(t)=\sum_{i \leq 0} a_{i}^{*} t^{i}, \quad g(t)=\sum_{i \geq 0} b_{i} t^{i}, \quad g^{*}(t)=\sum_{i \leq 0} b_{i}^{*} t^{i}
$$

and suppose that $f(t) g(t)=1, f^{*}(t) g^{*}(t)=1$.

Theorem 5.4. Let $\nu, \mu$ are partitions such that

$$
l(\nu)=q, l(\mu)=p, l\left(\nu^{\prime}\right)=r, l\left(\mu^{\prime}\right)=s .
$$


Then

$$
\begin{gathered}
\left|\begin{array}{cccc}
a_{\nu_{q}}^{*} & a_{\nu_{q}-1}^{*} & \ldots & a_{\nu_{q}-q-p+1}^{*} \\
\vdots & \vdots & \ddots & \vdots \\
a_{\nu_{1}+q-1}^{*} & a_{\nu_{1}+q-2}^{*} & \ldots & a_{\nu_{1}-p}^{*} \\
a_{\mu_{1}-q} & a_{\mu_{1}-q+1} & \ldots & a_{\mu_{1}+p-1} \\
\vdots & \vdots & \ddots & \vdots \\
a_{\mu_{p}-q-p+1} & a_{\mu_{p}-q-p+2} & \ldots & a_{\mu_{p}}
\end{array}\right| \\
=(-1)^{|\nu|+|\mu|}\left|\begin{array}{cccc}
b_{\nu_{s}^{\prime}}^{*} & b_{\nu_{s}^{\prime}-1}^{*} & \ldots & b_{\nu_{s}^{\prime}-s-r+1}^{*} \\
\vdots & \vdots & \ddots & \vdots \\
b_{\nu_{1}^{\prime}+s-1}^{*} & \tilde{b}_{\nu_{1}^{\prime}+s-2}^{*} & \ldots & b_{\nu_{1}^{\prime}-r}^{*} \\
b_{\mu_{1}^{\prime}-s} & b_{\mu_{1}^{\prime}-s+1} & \ldots & b_{\mu_{1}^{\prime}+r-1} \\
\vdots & \vdots & \ddots & \vdots \\
b_{\mu_{r}^{\prime}-s-r+1} & b_{\mu_{r}^{\prime}-s-r+2} & \ldots & b_{\mu_{r}^{\prime}}
\end{array}\right|
\end{gathered}
$$

Proof. Let us fix partitions $\mu$ and $\nu$. Then choose a natural number $m$ such that the following conditions are fulfilled

1) $p+q \leq m$

2) any index in the left hand side of the determinant and any index in the right hand side of the determinant is not grater then $m$. Equivalent conditions are $s+q-1 \leq m, r+p-1 \leq m$.

3) Elements $e_{i}^{*}, 1 \leq i \leq q+s-1, e_{i}, 1 \leq i \leq p+r-1$ algebraically independent.

Let now $\mathfrak{B} \subset \Lambda_{m}^{ \pm}$be the subring generated by $e_{i}^{*}, 1 \leq i \leq q+s-1, e_{i}, 1 \leq$ $i \leq p+r-1$. For any natural $k$ we have

$$
h_{k}=\left|\begin{array}{cccc}
e_{1} & \ldots & e_{k-1} & e_{k} \\
\vdots & \vdots & \ddots & \vdots \\
0 & \ldots & 1 & e_{1}
\end{array}\right|, \quad h_{k}^{*}=\left|\begin{array}{cccc}
e_{1}^{*} & 1 & \ldots & 0 \\
\vdots & \vdots & \ddots & \vdots \\
e_{k}^{*} & e_{k-1}^{*} & \ldots & e_{1}^{*}
\end{array}\right|
$$

therefore the elements of the determinant on the left hand side in the Lemma 5.3 belong to $\mathfrak{B}$. Consider a homomorphism $\varphi: \mathfrak{B} \longrightarrow \mathfrak{A}$ such that

$$
\varphi\left(e_{i}\right)=(-1)^{i} b_{i}, 1 \leq i \leq q+s-1 \varphi\left(e_{i}^{*}\right)=(-1)^{i} b_{i}^{*}, 1 \leq i \leq p+r-1
$$

according to our assumptions for $a_{i}, a_{i}^{*}, b_{j}, b_{j}^{*}$ the conditions (3) are satisfied. Therefore $\varphi\left(h_{i}\right)=a_{i}, \varphi\left(h_{i}^{*}\right)=a_{i}^{*}$. If we apply homomorphism $\varphi$ to the both sides of the equality from Lemma 5.3 we get the Theorem.

Now we are ready to construct a basis in the ring $\Lambda_{m, n}^{ \pm}$.

Definition 5.5. Let $(\lambda, \mu)$ be two sequences of non-increasing integers. Let us write the sequence $\mu$ in the form $\mu=\left(\tau_{1}, \ldots, \tau_{r}, 0, \ldots, 0-\nu_{s}, \ldots,-\nu_{1}\right)$, where $\nu, \tau$ are partitions. Let $\sigma$ be the sequence defined by the rule

$$
\left(\sigma_{1}, \ldots, \sigma_{l+p+k}\right)=\left(\nu_{1}^{\prime}, \ldots, \nu_{l}^{\prime}, \lambda_{1}, \ldots, \lambda_{p}, \tau_{1}^{\prime}, \ldots, \tau_{k}^{\prime}\right)
$$


where $p=\hat{l}(\lambda)$ and' means the conjugate partition. Let us define an element $K_{\lambda, \mu}$ of the ring $\Lambda_{m, n}^{ \pm}$by the formula

$$
K_{\lambda, \mu}=\operatorname{det}\left(a_{i j}\right), \text { where } a_{i j}=\left\{\begin{array}{l}
h_{\sigma_{i}+i-j}^{*}, 1 \leq i \leq l \\
H_{\sigma_{i}-i+j}, l<i \leq l+p \\
h_{\sigma_{i}-i+j}, l+p<i \leq l+p+k
\end{array},\right.
$$

and in all cases $1 \leq j \leq l+p+k$

Let us denote by $P(n, m)$ the set of pairs of sequences of non-increasing integers $(\lambda, \mu)$ and such that

$$
\hat{l}(\lambda) \leq m, \hat{l}(\mu) \leq n, \hat{l}(\lambda)-\hat{l}(\mu)=m-n
$$

Theorem 5.6. Let $(\lambda, \mu) \in P(m, n)$ then set of all $K_{\lambda, \mu}$ form a basis in the $\operatorname{ring} \Lambda_{m, n}^{ \pm}$.

Proof. We will use induction on $m n$. If $m n=0$, then either $m=0$, or $n=0$. If $m=0$, then $\hat{l}(\lambda)=0, \hat{l}(\mu)=n, h_{i}(\emptyset, y)=(-1)^{i} e_{i}(y), h_{i}^{*}=(-1)^{i} e_{i}^{*}, 1 \leq$ $i \leq n$. From the Theorem 5.4 and Lemma 2.9 it follows that

$$
K_{\emptyset, \mu}(y)=(-1)^{|\nu|+|\tau|} E_{\mu}(y) .
$$

This polynomials form a basis in $\Lambda_{n}^{ \pm}$again by Lemma 2.9. If $n=0$, then $\mu=\emptyset, \hat{l}(\lambda)=m$ and the statement follows from the Theorem 2.6.

Let now $n m>0$. Consider homomorphism

$$
\varphi_{m, n}: \Lambda_{m, n}^{ \pm} \longrightarrow \Lambda_{m-1, n-1}^{ \pm}, \quad \varphi\left(x_{m}\right)=\varphi\left(y_{n}\right)
$$

and on the other variables it acts identically. By inductive assumption polynomials $\varphi\left(K_{\lambda, \mu}\right)$ such that $(\lambda, \mu) \in X(m-1, n-1)$ form a basis in $\Lambda_{m-1, n-1}^{ \pm}$. Therefore in order to prove the Theorem we need to show that, polynomials $K_{\lambda, \mu}$ such that $\hat{l}(\lambda)=m, \hat{l}(\mu)=n$ form a basis of the kernel of the homomorphism $\varphi_{m, n}$. Actually it is enough to prove that

$$
K_{\lambda, \mu}=(-1)^{|\tau|+|\nu|} \prod_{j=1}^{n} \prod_{i=1}^{m}\left(1-\frac{y_{j}}{x_{i}}\right) E_{\lambda}\left(x_{1}, \ldots, x_{m}\right) E_{\mu}\left(y_{1} \ldots, y_{n}\right),
$$

This means that $K_{\lambda, \mu}$ are supercharacters of Kac modules up to a sign.

We have

$$
K_{\lambda, \mu} \Delta_{m}(x) \Delta_{n}(y)=\left\{K_{\lambda, \mu}(x, y) x^{\rho_{m}} y^{\rho_{n}}\right\}
$$

where as before $\{f(x, y)\}$ means alternation on the group $S_{m} \times S_{n}$. Applying alternation operation to the row number $l+1$ we come to equality

$$
K_{\lambda, \mu} \Delta(x) \Delta(y)=\left\{\prod_{j=1}^{n}\left(1-\frac{y_{j}}{x_{1}}\right) \tilde{K}_{\lambda, \mu}(x, y) x^{\rho_{m}} y^{\rho_{n}}\right\}
$$

where the determinant $\tilde{K}_{\lambda, \mu}(x, y)$ differs from the $K_{\lambda, \mu}$ only in the row number $l+1$ which is

$$
\left(x_{1}^{\lambda_{1}-l+1}, x_{1}^{\lambda_{1}-l+2}, \ldots, x_{1}^{\lambda_{1}+p+k}\right)
$$


Now let us multiply every column (starting from the first one ) of the determinant $\tilde{K}_{\lambda, \mu}(x, y)$ by $x_{1}$ and subtract it from the following column. Then using the equalities

$$
\begin{gathered}
h_{i-1}^{*}-x_{1} h_{i}^{*}=-x_{1} h_{i}^{*}\left(x_{2}, \ldots, x_{m}, y\right) \\
h_{i}-x_{1} h_{i-1}=h_{i}\left(x_{2}, \ldots, x_{m}, y\right)
\end{gathered}
$$

and expanding determinant along its row number $l+1$ we come to the equality

$$
K_{\lambda, \mu} \Delta_{m}(x) \Delta_{n}(y)=\left\{\prod_{j=1}^{n}\left(1-\frac{y_{j}}{x_{1}}\right) x_{1}^{\lambda_{1}} K_{\lambda^{(1)}, \mu}\left(x^{(1)}, y\right) x^{\rho_{m}} y^{\rho_{n}}\right\}
$$

where

$$
K_{\lambda^{(1)}, \mu}\left(x^{(1)}, y\right)=K_{\lambda_{2}, \ldots, \lambda_{m}, \mu}\left(x_{2}, \ldots x_{m}, y_{1}, \ldots, y_{n}\right)
$$

If we apply previous arguments $m$ times then we come to the equality

$$
K_{\lambda, \mu} \Delta_{m}(x) \Delta_{n}(y)=\left\{\prod_{j=1}^{n} \prod_{i=1}^{m}\left(1-\frac{y_{j}}{x_{i}}\right) x_{1}^{\lambda_{1}} \ldots x_{m}^{\lambda_{m}} K_{\emptyset, \mu}(\emptyset, y) x^{\rho_{m}} y^{\rho_{n}}\right\}
$$

But we have already proved that

$$
K_{\emptyset, \mu}(\emptyset, y)=(-1)^{|\mu|+|\nu|} E_{\mu}(y)
$$

And Theorem is proved.

Corollary 5.7. Let $(\lambda, \mu) \in P(m, n)$ and $\mu$ is a partition then set of all $K_{\lambda, \mu}$ form a basis in the ring $\Lambda_{m, n}^{+y}$.

Proof. It is clear that if $\mu$ is a partition then $K_{\lambda, \mu} \in \Lambda_{m, n}^{+y}$. So it is enough to prove that such elements linearly generated the ring $\Lambda_{m, n}^{+y}$. Let us prove it induction on $m n$. If $m=0$, then we already proved that $K_{\emptyset, \mu}(y)=$ $(-1)^{|\mu|} E_{\mu}(y)$ and therefore linearly generate $\Lambda_{n}$. If $n=0$, then as before the statement follows from the Theorem 2.6. If $m n>0$, then considering homomorphism $\varphi_{m, n}$ and applying inductive assumption we see that it is enough to prove that the kernel of the homomorphism $\varphi_{m, n}$ is a linear span of the elements $K_{\lambda, \nu}$ such that $\hat{l}(\lambda)=m, \hat{l}(\mu)=n$. But in this case $K_{\lambda, \nu}$ can be written in the form (4) and therefore they linearly generate the kernel.

Corollary 5.8. Let $(\lambda, \mu) \in P(m, n)$ and $\lambda, \mu$ are partitions then set of all $K_{\lambda, \mu}$ form a basis in the ring $\Lambda_{m, n}$.

Proof. The proof can be given exactly in the same manner as the proof of the previous corollary.

Remark 5.9. It is not difficult to show that there is a bijection between set of partitions $(\lambda, \mu) \in P(m, n)$ and the set of partitions $\nu$ such that $\nu_{m+1} \leq$ $n$ and the definition of $K_{\lambda, \mu}$ coincides with the Jacobi-Trudy formula for 
supersymmetric Schur functions. So in particular our formulae are natural generalisations of Jacobi-Trudy formulae.

Now let us show that our $K_{\lambda, \mu}$ are particular case of Euler supercharacters formulae.

Let us define for $(\lambda, \mu) \in P(m, n)$ two sets of natural numbers

$$
D_{+}=[1, p] \times[1, n], \quad D_{-}=[p+1, m] \times[1, q]
$$

where $p=\hat{l}(\lambda), q=\hat{l}(\mu)$. Set also $x^{\rho_{m}}=x_{1}^{m-1} \ldots x_{m}^{0}, y^{\rho_{n}}=y_{1}^{n-1} \ldots y_{n}^{0}$.

Proposition 5.10. The following equality is valid

$$
\begin{gathered}
K_{\lambda, \mu} \Delta(x) \Delta(y)= \\
=(-1)^{a}\left\{\prod_{(i, j) \in D_{+}}\left(1-\frac{y_{j}}{x_{i}}\right) \prod_{(i, j) \in D_{-}}\left(1-\frac{x_{i}}{y_{j}}\right) x_{1}^{\lambda_{1}} \ldots x_{p}^{\lambda_{p}} y_{1}^{\mu_{1}} \ldots y_{q}^{\mu_{q}} x^{\rho_{m}} y^{\rho_{n}}\right\}
\end{gathered}
$$

where $a=|\tau|+|\nu|$.

Proof. Let us use induction on $p$. We start with minimal value of $p$. If $n \geq m$, then minimal value of $p$ is 0 . If $n<m$, the minimal value of $p$ is $m-n$. In the first case $q=n-m$. By Lemma (5.4)

$$
K_{\emptyset, \mu}(x, y)=(-1)^{|\nu|+|\tau|} K_{\mu, \emptyset}(y, x)
$$

. The determinant on the right hand side contains $h_{i}^{*}(y, x)$ and $h_{i}(y, x)$. The minimal index in the first case is $\nu_{s}^{\prime}-s-r+1$, in the second case is $\tau_{r}^{\prime}-r-s+1$. It follows from the definition that $h_{i}(y, x)=H_{i}(y, x)$, и $h_{i}^{*}(y, x)=(-1)^{n+1} \frac{x_{1} \ldots x_{m}}{y_{1} \ldots n} H_{m-n-i}(y, x)$, if $i>m-n$. By our assumptions $s+r \leq q=n-m$, therefore both of minimal indexes strictly grate then $m-n$ so all the small letters $h, h^{*}$ can be replaced by the capital letters $H$. Therefore by Lemma 3.5 the statement of the Theorem is true in this case. In the second case $m>n, p=m-n, q=0$ and statement of the Theorem follows from the Lemma 3.5.

Let $p>0$, then $m>0$ so, applying equality (5) and taking into account that $\tau_{1}+\nu_{1} \leq p+n-m=(p-1)+n-(m-1)$ we can reduce the proof to the case $p-1$ and use inductive assumption.

\section{EXAMPLE}

Let us consider the most simple non-trivial example when $m=n=1$. In this case

$$
\Lambda_{1,1}^{ \pm}=\left\{f \in \mathbb{Z}\left[x^{ \pm 1}, y^{ \pm 1} \mid x \frac{\partial f}{\partial x}+y \frac{\partial f}{\partial y} \in(x-y)\right\}\right.
$$

and

$$
P(1,1)=\{(\lambda, \mu), \lambda, \mu \in \mathbb{Z}\} \cup\{\emptyset\}
$$

Let us write down the corresponding elements of the basis. 
If $\mu=r>0$, then

$$
K_{\lambda, \mu}=\left|\begin{array}{cccc}
H_{\lambda} & H_{\lambda+1} & \ldots & H_{\lambda+r} \\
h_{0} & h_{1} & \ldots & h_{r} \\
\vdots & \vdots & \ddots & \vdots \\
h_{1-r} & \ldots & h_{0} & h_{1}
\end{array}\right|=-\left(1-\frac{y}{x}\right) x^{\lambda} y^{\mu}
$$

If $\mu=-s<0$, then

$$
K_{\lambda, \mu}=\left|\begin{array}{cccc}
h_{1}^{*} & h_{0}^{*} & \ldots & h_{1-s}^{*} \\
\vdots & \vdots & \ddots & \vdots \\
h_{s}^{*} & h_{s-1}^{*} & \ldots & h_{0}^{*} \\
H_{\lambda-s} & H_{\lambda+1-s} & \ldots & H_{\lambda}
\end{array}\right|=-\left(1-\frac{y}{x}\right) x^{\lambda} y^{\mu}
$$

and if $\mu=0$, then $K_{\lambda, 0}=H_{\lambda}=\left(1-\frac{y}{x}\right) x^{\lambda}$ and $K_{\emptyset}=1$.

The ring $\Lambda_{1,1}^{ \pm}$is isomorphic to the ring $U_{1,1}^{ \pm}$generated by $u_{i}, v_{i}, t, i \in \mathbb{Z}$ such that $u_{0}=v_{0}=1, u_{i}=v_{i}=0, i<0$ subject to the relations

$$
\left|\begin{array}{cc}
w_{i} & w_{i+1} \\
w_{j} & w_{j+1}
\end{array}\right|=0, \quad w_{i}=u_{i}-t v_{i}, \quad i, j \in \mathbb{Z}
$$

and the corresponding homomorphism has the form

$$
\varphi: U_{1,1}^{ \pm} \longrightarrow \Lambda_{1,1}^{ \pm}, \varphi\left(u_{i}\right)=h_{i}, \varphi\left(v_{i}\right)=h_{i}^{*}, \varphi(t)=\frac{y}{x}
$$

In the case $\Lambda_{1,1}^{+y}$ the basis forms $K_{\lambda, \mu}$ such that $\mu \geq 0$ and $K_{\emptyset}$.

The ring $\Lambda_{1,1}^{+y}$ is isomorphic to the ring $U_{1,1}^{+}$generated by $u_{i}, v_{i}, i \in \mathbb{Z}$ such that $u_{0}=1, u_{i}=v_{i}=0, i<0$ subject to the relations

$$
\left|\begin{array}{ll}
w_{i} & w_{i+1} \\
w_{j} & w_{j+1}
\end{array}\right|=0, \quad w_{i}=u_{i}-v_{i}, \quad i, j \in \mathbb{Z}
$$

and the corresponding homomorphism has the form

$$
\varphi: U_{1,1}^{ \pm} \longrightarrow \Lambda_{1,1}^{+}, \varphi\left(u_{i}\right)=h_{i}, \varphi\left(v_{i}\right)=\frac{y}{x} h_{i}^{*}, \varphi(t)=\frac{y}{x}
$$

In the case $\Lambda_{1,1}^{+y}$ the basis forms $K_{\lambda, \mu}$ such that $\lambda, \mu \geq 0$ and $K_{\emptyset}$.

The ring $\Lambda_{1,1}$ is isomorphic to the ring $U_{1,1}$ generated by $u_{i} \in \mathbb{Z}$ such that $u_{0}=1, u_{i}=0, i<0$ subject to the relations

$$
\left|\begin{array}{ll}
u_{i} & u_{i+1} \\
u_{j} & u_{j+1}
\end{array}\right|=0, \quad i \in \mathbb{Z}_{>0}
$$

and the corresponding homomorphism has the form

$$
\varphi: U_{1,1} \longrightarrow \Lambda_{1,1}, \varphi\left(u_{i}\right)=h_{i} .
$$

\section{ACNOWledgements}

I am grateful to A.I. Molev and M.L. Nazarov for useful discussions.

This work has been funded by the Russian Academic Excellence Project '5100' and by the Russian Ministry of Education and Science (grant 1.492.2016/1.4). 


\section{REFERENCES}

[1] J. Brundan Kazhdan-Lusztig polynomials and character formulae for the Lie superalgebra $\mathfrak{g l}(m \mid n)$. J. Amer. Math. Soc. 16 (2003), no. 1, 185-231.

[2] C.J. Cummins, R.C. King, Composite Young diagram, supercharacters of $U(M / N)$ and modification rules. J. Phys. A : Math. Gen. 20 (1987) 3121-3133.

[3] W. Fulton, J. Harris, Representation Theory. A First Course. Grad. Texts in Math., Reading in Mathematics, vol.129, Springer-Verlag, New York 1991.

[4] F.R. Gantmacher The Theory of Matrices. vol.1, Chelsea Publishing Company, New York 68,1959, $374 \mathrm{pp}$.

[5] C. Gruson, V. Serganova Cohomology of generalized super grassmannians and character formula for basic classical Lie superalgebras. In preparation.

[6] C.Hoyt, S. Reif Duflo-Sergenova functor and Grothendieck rings of Lie superalgebras, arXiv: 1612.05815 (2016).

[7] I. Kantor, I. Trishin The algebra of polynomial invariants of the adjoint representation of the Lie superalgebra $g l(n, m)$. Communication in algebra, 25(7), 1997, 2039-2070.

[8] H.M. Khudaverdian, Th.Th. Voronov Berezinians, exterior powers and recurrent sequences. Lett. Math. Phys. 74 (2005), no. 2, 201-228.

[9] I. Macdonald Symmetric functions and Hall polynomials. 2nd edition, Oxford Univ. Press, 1995.

[10] E. Moens Supersymmetric Schur functions and Lie superalgebra representations. PhD Thesis, Universitet Gent, 2007. https:/ biblio.ugent.be/publicatin/469926

[11] E.M. Moens and J. Van der Jeugt, Composite supersymmetric S-functions and characters of $\mathrm{gl}(\mathrm{m} / \mathrm{n})$ representations. Bulg. J. Phys. 33 (s2) (2006), 251-268.

[12] V. Serganova Characters of irreducible representations of simple Lie superalgebras. Proc. Internat. Congr. Math., Vol. II (Berlin, 1998), Extra Vol. II, 1998, 583-593.

[13] Sergeev, Alexander N.; Veselov, Alexander P.Grothendieck rings of basic classical Lie superalgebras. Ann. of Math. (2) 173 (2011), no. 2, 663-703.

Department of Mathematics, Saratov State University, Astrakhanskaya 83, Saratov 410012, Russia and National Research University Higher School of Economics, Russian Federation.

E-mail address: SergeevAN@info.sgu.ru 